\title{
The Jones-Ray Effect Is Not Caused by Surface-Active Impurities
}

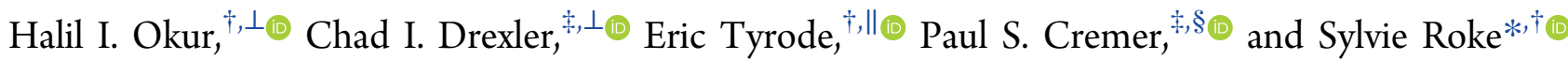

${ }^{\dagger}$ Laboratory for Fundamental BioPhotonics (LBP), Institute of Bioengineering (IBI) and Institute of Materials Science (IMX), School of Engineering (STI), and Lausanne Center for Ultrafast Science (LACUS), École Polytechnique Fédérale de Lausanne (EPFL), CH-1015 Lausanne, Switzerland

${ }^{\ddagger}$ Department of Chemistry and ${ }^{\S}$ Biochemistry and Molecular Biology, The Pennsylvania State University, University Park, Pennsylvania 16802, United States

"Department of Chemistry, School of Engineering Sciences in Chemistry, Biotechnology and Health, KTH Royal Institute of Technology, SE-10044 Stockholm, Sweden

\section{Supporting Information}

ABSTRACT: Pure aqueous electrolyte solutions display a minimum in surface tension at concentrations of $2 \pm 1 \mathrm{mM}$. This effect has been a source of controversy since it was first reported by Jones and Ray in the 1930s. The Jones-Ray effect has frequently been dismissed as an artifact linked to the presence of surface-active impurities. Herein we systematically consider the effect of surface-active impurities by purposely adding nanomolar concentrations of surfactants to dilute electrolyte solutions. Trace amounts of surfactant are indeed found to decrease the surface tension and influence the surface chemistry. However, surfactants can be removed by repeated aspiration and stirring cycles, which eventually deplete the surfactant from solution, creating a pristine surface. Upon following this cleaning procedure, a reduction in the surface tension by millimolar concentrations of salt is still observed. Consequently, we demonstrate that the Jones-Ray effect is not caused by surfaceactive impurities.

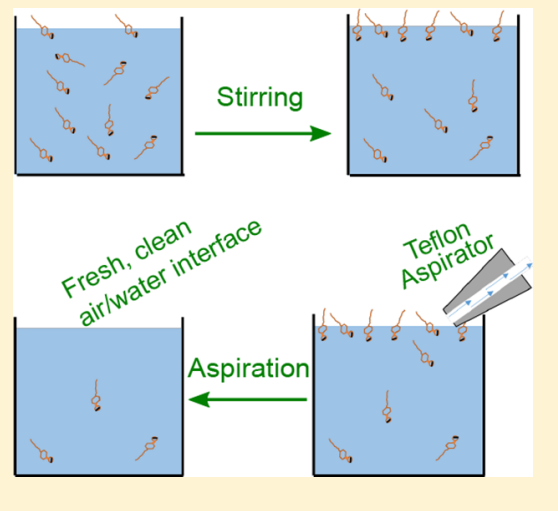

A queous interfaces are important for a plethora of biological, chemical, and physical processes. ${ }^{1}$ Quantifying interfacial properties has been a major aim in the last few decades, and thus various experimental and simulation approaches have been introduced. ${ }^{2-7}$ Planar extended interfaces are most offen employed in experimental studies. For such surfaces, there is the experimental complication of having a small surface-to-volume ratio that gives rise to possible undetectable artifacts in the experimental data. ${ }^{8}$ This is especially true when the contaminants are surfactants, which can be far more surface-active than the materials under study (i.e., salts).

The influence of impurities is a topic that has long been a source of concern in the interfacial chemistry community. ${ }^{9-16}$ The discussion was recently refueled by theoretical studies ${ }^{17,18}$ that suggested that surfactant impurities may be responsible for many surface chemical observations and, most notably, for the Jones-Ray effect. In 1937, Jones and Ray reported the surface tension of aqueous $\mathrm{KCl}$ solutions at very low ionic strength. ${ }^{19}$ They measured that the surface tension gradually decreases, reaching a surface tension minimum at $2 \pm 1 \mathrm{mM}$. Above 2 $\mathrm{mM}$, the surface tension gradually increases again. This observation has been a topic of much debate because the result is counterintuitive and the change in surface tension is small. Impurities might, therefore, be a plausible cause, which would obviate the need for a discussion about the underlying mechanism.
The Jones-Ray effect has been recently reproduced. ${ }^{20-22}$ Figure 1 shows the change in surface tension $(\Delta \gamma)$ as a function of $\mathrm{NaCl}$ concentration in $\mathrm{H}_{2} \mathrm{O}$ (blue) and in $\mathrm{D}_{2} \mathrm{O}$ (red), as measured by the Wilhelmy plate method (illustrated in Figure 1A). For both $\mathrm{H}_{2} \mathrm{O}$ and $\mathrm{D}_{2} \mathrm{O}$, the surface tension $(\gamma)$ decreases at low electrolyte concentrations. For $\mathrm{NaCl}$, in $\mathrm{H}_{2} \mathrm{O}$, there is a minimum near $2 \pm 1 \mathrm{mM}\left(\Delta \gamma=-0.24 \mathrm{~mJ} / \mathrm{m}^{2}\right)$. The minimum in surface tension occurs at $\sim 14 \mathrm{mM}(\Delta \gamma=-0.29$ $\mathrm{mJ} / \mathrm{m}^{2}$ ) for $\mathrm{D}_{2} \mathrm{O}$. These observations show that small changes in the surface tension of dilute salt solutions can be measured. Moreover, the minimum in the surface tension of the $\mathrm{H}_{2} \mathrm{O}$ /air interface appears in the same concentration range as in the original measurements of Jones and Ray. $\mathrm{D}_{2} \mathrm{O}$ solutions, on the contrary, display a minimum at a very different $(\sim 14 \mathrm{mM})$, but still low, concentration. This difference suggests that solvention interactions play a major role in determining the freeenergy difference between the surface and the bulk (rather than the ion-surface interaction, as in the high salt concentration range). Combining these measurements ${ }^{21}$ with elastic second-harmonic scattering, reflection UV resonant second-harmonic generation, and modeling, ${ }^{20,23}$ we concluded that the Jones-Ray effect is not an experimental artifact and relates to the interactions of ions with water. We proposed that

Received: September 25, 2018

Accepted: November 6, 2018

Published: November 6, 2018 

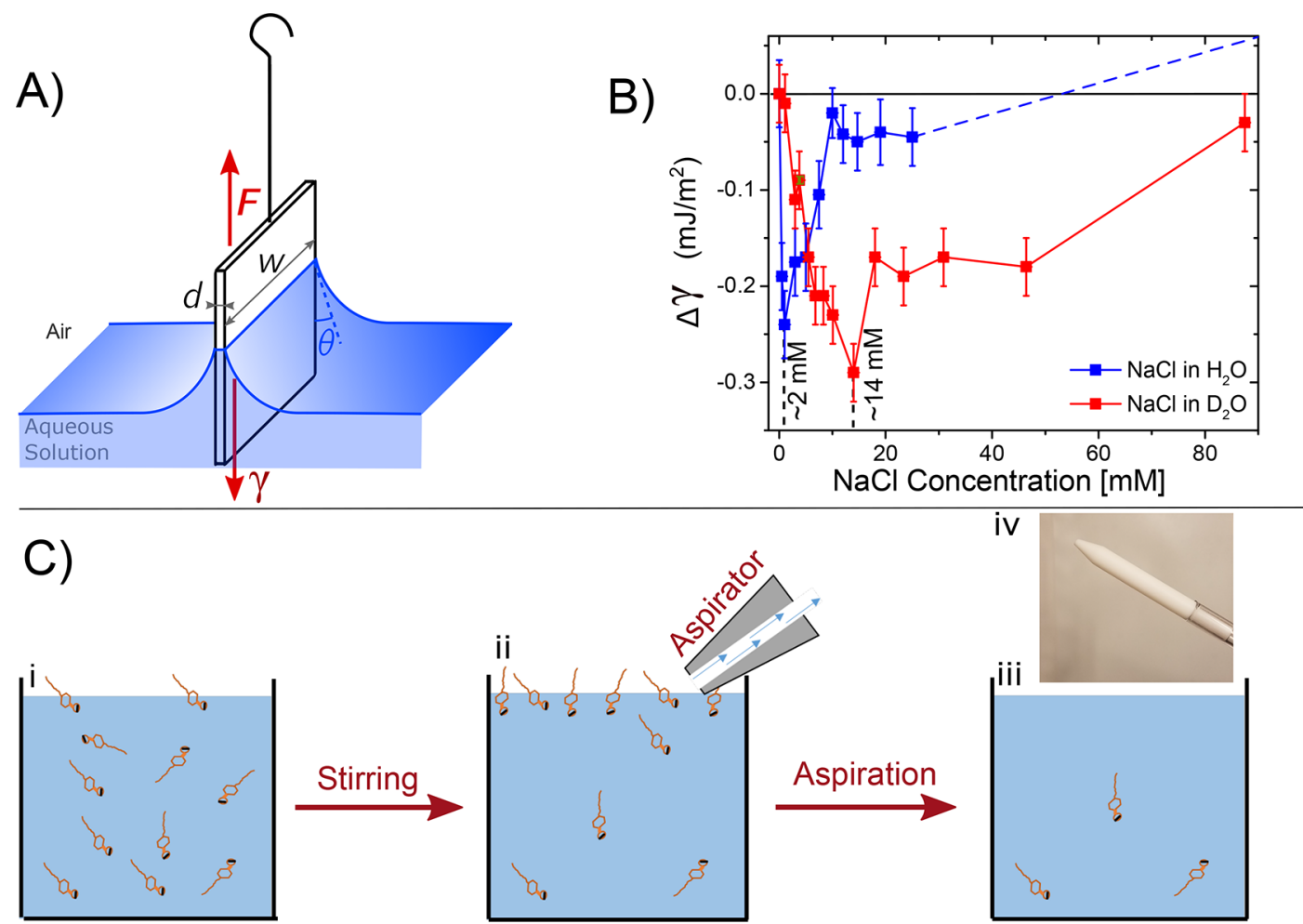

Figure 1. Wilhelmy plate measurement of $\mathrm{NaCl}$ added to $\mathrm{H}_{2} \mathrm{O}$ and $\mathrm{D}_{2} \mathrm{O}$. (A) Illustration of the Wilhelmy plate method. (B) Surface tension difference $(\Delta \gamma)$ between the salt solution and neat $\mathrm{H}_{2} \mathrm{O}\left(\gamma=72.9 \mathrm{~mJ} / \mathrm{m}^{2} @ 20^{\circ} \mathrm{C}\right)$, and $\mathrm{D}_{2} \mathrm{O}\left(\gamma=72.7 \mathrm{~mJ} / \mathrm{m}^{2}\right)$ measured as a function of $\mathrm{NaCl}$ concentration for solutions in $\mathrm{H}_{2} \mathrm{O}$ (blue) and in $\mathrm{D}_{2} \mathrm{O}$ (red). The red and blue lines connect the data points, and the dashed blue line indicates the surface tension increase based on literature. ${ }^{24}$ (C) Interfacial cleaning protocol for removing trace impurities of surfactants. (i-iii) Illustrations for the steps of stirring and aspiration cycles and (iv) a photograph of the Teflon aspirator.
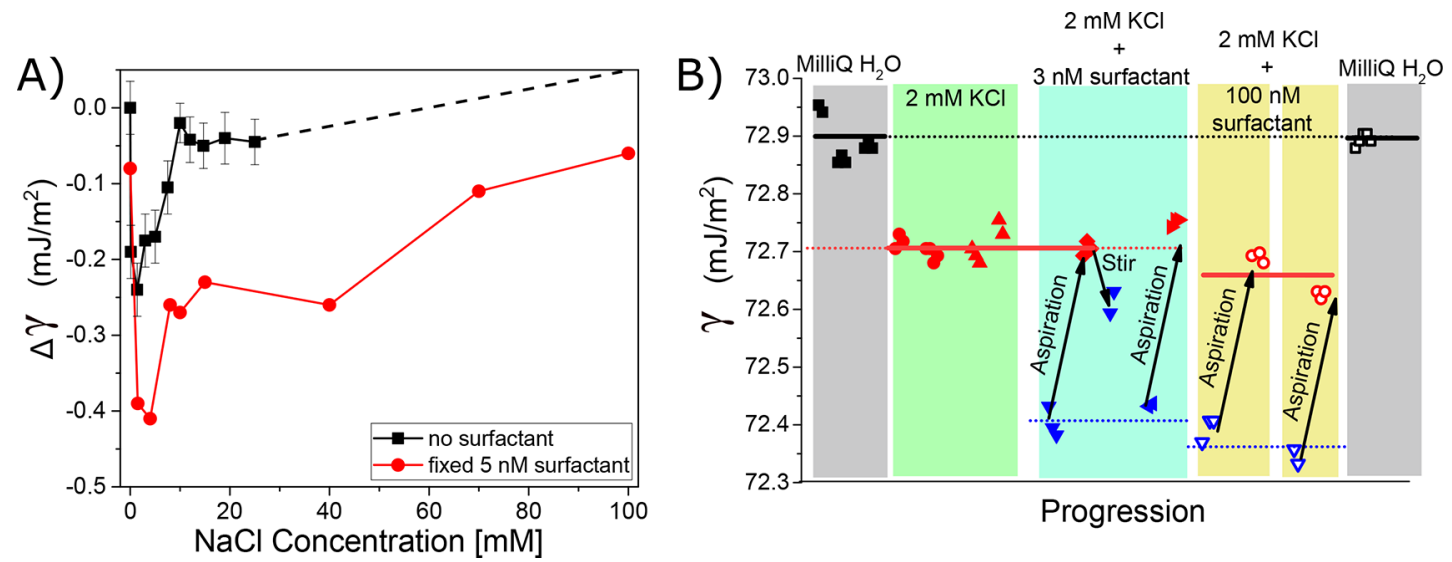

Figure 2. Effect of surfactant impurities on the surface tension of salt solution. (A) Surface tension data of a salt concentration series for NaCl added to ultrapure water (black trace) $)^{20,21}$ and a concentration series of $\mathrm{NaCl}$ added to a solution of $5 \mathrm{nM} \mathrm{NaDBS}$. (B) Surface tension values for different aqueous solutions and aspiration/stirring cycles: ultrapure water (gray areas), $2 \mathrm{mM}$ of $\mathrm{KCl}$ added to ultrapure water with stirring/ aspiration (green), and 3 (100) $\mathrm{nM} \mathrm{NaDBS}$ added to $2 \mathrm{mM} \mathrm{KCl}$ solution shown in cyan (yellow) area. Stirring/aspiration brings back the surface tension value to the pure $2 \mathrm{mM} \mathrm{KCl}$ solution value but not to the value of pure water (gray). Blue triangles (open and filled) show data points after stirring and before aspiration, whereas red and black (open and filled) data points show measurement after aspiration. Note that measurements of different sample solutions with identical composition are indicated with differently shaped data points. Thus the former measures the impurity contamination, and the latter measures the surfactant-free interface.

the surface tension minimum originates from bulk ion-induced water-water correlations rather than ion adsorption at the interface. As ions are added to water, the hydrogen-bond network of water responds to the collective electrostatic field of ions by increasing its orientational order. This increase in orientational order in the bulk solution gives rise to an entropic penalty, which causes a reduction in the surface tension. ${ }^{21}$
Recent computational studies ${ }^{17,18}$ have suggested that the surface tension changes found in Figure 1B are caused by the presence of minute, undetectably small nanomolar concentrations of surfactants. In these studies, it is argued that saltinduced screening enhances the impurity adsorption at the interface, ${ }^{17,18}$ which leads to a depression in the surface tension. Although the question still remains as to how this 
could result in around an order of magnitude difference in salt concentration between $\mathrm{H}_{2} \mathrm{O}$ and $\mathrm{D}_{2} \mathrm{O}$, the calculated results open up three questions that we investigate here:

- Can minute amounts of surfactants influence the surface tension and thus the surface chemistry of salt solutions?

- Is this mechanism responsible for the Jones-Ray effect?

- Does it cast doubt over all surface tension measurements?

To answer these questions, we have repeated the surface tension measurements in Figure 1 with and without the addition of nanomolar concentrations of a negatively charged surfactant and adopted a simple but effective surface-cleaning approach, which consists of repeatedly aspirating the surface before performing the measurements. The method is inspired by a procedure frequently used to prepare clean Langmuir monolayers $^{25}$ as well as in the removal of surface-active contaminants from soluble surfactant solutions. ${ }^{13,26}$ An aspirator is a small Teflon tip connected to a vacuum pump (Figure 1C,iv) that can be used to remove the topmost portion of the aqueous solution including the surface-adsorbed molecules. By combining the aspiration procedure with stirring of the aqueous solution, it becomes possible to accelerate the interfacial adsorption of surfactants found in trace amounts in the bulk, which can then be removed, eventually depleting the amount of surfactants in solution. The process is illustrated in Figure 1C.

Figure 2 shows the change in surface tension compared to neat $\mathrm{H}_{2} \mathrm{O}(\Delta \gamma)$ as a function of $\mathrm{NaCl}$ concentration in $\mathrm{H}_{2} \mathrm{O}$ (black) and in $\mathrm{H}_{2} \mathrm{O}$ to which $5 \mathrm{nM}$ of sodium dodecylbenzenesulfonate (NaDBS) was added (red). These Wilhelmy plate measurements show minima in both curves at the same concentration, $\sim 2 \mathrm{mM}$, but with significantly different values: $\Delta \gamma=-0.24 \mathrm{~mJ} / \mathrm{m}^{2}$ at $\sim 2 \mathrm{mM} \mathrm{NaCl}$ without $\mathrm{NaDBS}$ and $\Delta \gamma=$ $-0.41 \mathrm{~mJ} / \mathrm{m}^{2}$ at $1-4 \mathrm{mM} \mathrm{NaCl}$ with $5 \mathrm{nM}$ of $\mathrm{NaDBS}$ added. This shows that surfactants can indeed decrease the surface tension minimum, and the mechanism could well be that the presence of ions enhances the local concentration of surfactants at the interface, thereby giving rise to an ion concentration-dependent minimum in the interfacial tension. ${ }^{17,18}$

Is the enhancement of surfactant impurities by electrolytes at the interface also an explanation for the Jones-Ray effect? To test this question, we have first measured again the Jones-Ray effect but this time with stirring/aspiration cycles. Figure $2 \mathrm{~B}$ shows the surface tension value of "ultrapure" water, 18.1 M $\Omega$. $\mathrm{cm}$ (gray zone), and the surface tension values for a solution of $2 \mathrm{mM} \mathrm{KCl}$ that has been subject to three stirring/aspiration cycles. It can be seen that the surface tension values remain $\sim 0.2 \mathrm{mN} / \mathrm{m}$ lower compared with pure water and remain identical for all measurements. This value coincides with the minimum found in Figure 1b. Next, we measured a solution with the same salt concentration with $3 \mathrm{nM} \mathrm{NaDBS}$ added. A clear reduction in the surface tension, $(\gamma)$, is observed compared to pure water and the $2 \mathrm{mM} \mathrm{KCl}$ solutions. As anticipated, this extra decrease in the surface tension values can be removed by aspiration of the air/water interface. After aspiration and upon continued stirring, the surface tension drops again on a time scale of $30 \mathrm{~min}$. Stirring accelerates the adsorption of surfactant to the surface, as diffusion for $n M$ surfactant concentrations is slow in the absence of convection. Aspirating again moves the surface tension back up to the initial value of the $2 \mathrm{mM} \mathrm{KCl}$ solutions but not to that of neat water. The same behavior is observed if $100 \mathrm{nM}$ of $\mathrm{NaDBS}$ is added. Repeated stirring/aspiration cycles will eventually deplete the surface-active species from solution, ${ }^{13,26}$ bringing the surface tension minimum close to the original value of a pure $2 \mathrm{mM} \mathrm{KCl}$ solution. This shows that charged surfactants can indeed influence the surface tension of aqueous solutions, but a surface-cleaning procedure can easily remove such impurity effects. To test the influence of the type of salt and the general applicability of the method, we repeated the results for aqueous $\mathrm{NaCl}$ solution at a different laboratory on another continent; see Figure S1. We thus conclude that nanomolar concentrations of surfactants in solution can decrease the surface tension minimum observable for salt solutions, as predicted by recent simulations studies. ${ }^{17,18}$ However, stirring/ aspiration cycles can remove the surfactants from the system but do not lead to a disappearance of the Jones-Ray effect. This means that surface-active contaminants are not the cause of the Jones-Ray effect. Rather, ions induce small structural changes to the hydrogen-bond network of water that result in a minimum of the surface tension, as explained in detail in ref 21 .

In summary, we have investigated the possibility that surfactant-active impurities influence surface chemistry and surface tension measurements in particular. We found a persistent surface tension minimum $(\Delta \gamma \approx-0.2 \pm 0.05 \mathrm{mN} /$ $\mathrm{m})$ at $2 \pm 1 \mathrm{mM}$ ionic strength for electrolyte aqueous solution/air interfaces, even with repeated stirring/aspiration cycles. This clearly indicates that an interplay of electrolytes and water is the underlying mechanism for the Jones-Ray effect rather than an ion-induced enhanced concentration of surfactants in the interfacial region. By contrast, when nanomolar concentrations of charged surfactants are present, the surface tension minimum is still observed at the same concentration but drops to a lower value $(\Delta \gamma=-0.41 \mathrm{mN} / \mathrm{m}$ for $\mathrm{NaCl}$ ). By removing the surfactant-rich interfacial region using stirring-aspiration cycles, the surface tension increases to that of the neat electrolyte solution/air interface. This shows that surface-active impurities can serve as an artifact to surface tension and quite possibly to many other surface chemistry measurements but are not the origin of the Jones-Ray effect. The Jones-Ray effect stems from an interplay between ions and water, whereby the ions induce tiny modifications in the hydrogen-bond structure of water.

\section{MATERIALS AND METHOD}

Chemicals. $\mathrm{NaCl}$ (Abcr and Sigma-Aldrich 99.999\%) and $\mathrm{KCl}$ (Acros Chemicals, 99.999\%) were first baked at $600{ }^{\circ} \mathrm{C}$ for $2 \mathrm{~h}$ prior to use. NaDBS (Sigma-Aldrich, Pharmaceutical secondary standard) is employed as the negatively charged surfaceactive impurity. A NANOpure Ultrapure Water System (Barnstead) and a Milli-Q-UF-Plus instrument (Millipore) were employed to obtain $18.1 \mathrm{M} \Omega \cdot \mathrm{cm}$ water to prepare light water $\left(\mathrm{H}_{2} \mathrm{O}\right)$ samples. Samples were prepared in glassware that was cleaned by two different methods, and identical results were achieved. In the first (Figure 1, Figure S1), the glassware was cleaned with piranha $\left(3: 1 \mathrm{H}_{2} \mathrm{SO}_{4}(95-98 \%\right.$, Sigma) and $\mathrm{H}_{2} \mathrm{O}_{2}(30 \%$, Macron Fine Chemicals) and cleaned and rinsed with copious amounts of ultrapure water prior to use. In the second (Figure 2), a cleaning solution was prepared with Deconex by 1:20 dilution, and glassware were filled with this solution, sonicated for $1 \mathrm{~h}$, and washed and rinsed at least 10 times with Milli-Q water. The surfactant solutions at nanomolar concentrations were prepared as follows: A 10 $\mathrm{mL}$ stock solution of surfactant at its critical micelle 
concentration was prepared, and 1:100 dilutions were performed until the desired surfactant concentration was achieved in previously cleaned $100 \mathrm{~mL}$ volumetric glassware. Furthermore, we used negatively charged surfactants to minimize any potential adsorption to negatively charged glass surface. Then, the desired sample solutions are measured in jacketed glass containers with a surface area of $\sim 50 \mathrm{~cm}^{2}$ and a solution volume of $50 \mathrm{~mL}$.

Surface Tension Measurements. Surface tension measurements were performed using the Wilhelmy plate method. The Wilhelmy plate method, ${ }^{8}$ as illustrated in Figure 1A, uses a vertically suspended Pt plate (Biolin Scientific) with a perimeter (l), which is pulled out of the electrolyte solution toward the air. The force $(F)$ acting on this plate was measured with a microbalance, and it was correlated to the surface tension $(\gamma)$ by the following equation

$$
\gamma=\frac{F}{l \cos (\theta)}
$$

Here $\theta$ is the contact angle between Pt and water. $\theta$ is assumed to have a value of $\theta=0$ based on literature. ${ }^{27}$ With $\theta=0^{\circ}$, the measurement accuracy of the method is $\sim 0.1 \%{ }^{8}$ For a more detailed description of surface tension, see ref 21 .

To measure small changes in the surface tension, care needs to be taken to eliminate possible surface-active contaminants. To help eliminate this effect, all solutions were stored in closed glass containers with glass caps. The glassware was cleaned in a piranha solution $\left(3: 1 \mathrm{H}_{2} \mathrm{SO}_{4} / \mathrm{H}_{2} \mathrm{O}_{2}\right)$ or with Deconex solutions, as described above, before use. Moreover, sample solutions were freshly prepared just prior to measurements with degassed ultrapure water and with different batches of highly pure $\mathrm{NaCl}$ or $\mathrm{KCl}$ salt $(>99.999 \%$, the highest commercially available). The salts were baked at $600{ }^{\circ} \mathrm{C}$ for $2 \mathrm{~h}$ prior to use to remove potential organic contaminants. Environmental influences including any possible dust contamination, vibrational instability, and temperature were minimized to the extent possible. The measurements were performed in a jacketed beaker, and the temperature was monitored to remain at $20 \pm 0.05{ }^{\circ} \mathrm{C}$ over the course of the experiments. Moreover, personal protective equipment including nitrile gloves and clean-room-compatible garments were worn at all the times. The Pt Wilhelmy plate was first cleaned with a piranha solution or with Nochromix (Sigma-Aldrich) and then rinsed with copious amounts of Milli-Q water to eliminate organic impurities from prior measurements.

\section{ASSOCIATED CONTENT}

\section{S Supporting Information}

The Supporting Information is available free of charge on the ACS Publications website at DOI: 10.1021/acs.jpclett.8b02957.

Additional surface tension measurements and Figure S1 (PDF)

\section{AUTHOR INFORMATION}

\section{Corresponding Author}

*E-mail: sylvie.roke@epfl.ch.

\section{ORCID $\odot$}

Halil I. Okur: 0000-0002-2492-1168

Chad I. Drexler: 0000-0002-9989-968X

Eric Tyrode: 0000-0003-1221-0227
Paul S. Cremer: 0000-0002-8524-0438

Sylvie Roke: 0000-0002-6062-7871

\section{Author Contributions}

${ }^{\perp}$ H.I.O. and C.I.D. contributed equally.

\section{Notes}

The authors declare no competing financial interest.

\section{ACKNOWLEDGMENTS}

H.I.O. and S.R. thank the Julia Jacobi Foundation, the Swiss National Science Foundation, and the European Research Council (grant number 616305) for financial support. E.T. acknowledges the Swedish Foundation for Strategic Research (SSF-FFL-5 program) and the Swedish Research Council (V.R.) for financial support. C.I.D. and P.S.C. were supported by the National Science Foundation (CHE-1709735).

\section{REFERENCES}

(1) Chaffey, N.; Alberts, B.; Johnson, A.; Lewis, J.; Raff, M.; Roberts, K.; Walter, P. Molecular biology of the cell. 4th edn. Ann. Bot. 2003, 91, 401-401.

(2) Zaera, F. Probing Liquid/Solid Interfaces at the Molecular Level. Chem. Rev. 2012, 112, 2920-2986.

(3) Bain, C. D. Studies of adsorption at interfaces by optical techniques: Ellipsometry, second harmonic generation and sumfrequency generation. Curr. Opin. Colloid Interface Sci. 1998, 3, 287292.

(4) Miranda, P. B.; Shen, Y. R. Liquid Interfaces: A Study by SumFrequency Vibrational Spectroscopy. J. Phys. Chem. B 1999, 103, 3292-3307.

(5) Perera, P. N.; Fega, K. R.; Lawrence, C.; Sundstrom, E. J.; Tomlinson-Phillips, J.; Ben-Amotz, D. Observation of water dangling $\mathrm{OH}$ bonds around dissolved nonpolar groups. Proc. Natl. Acad. Sci. U. S. A. 2009, 106, 12230-12234.

(6) Petersen, P. B.; Johnson, J. C.; Knutsen, K. P.; Saykally, R. J. Direct experimental validation of the Jones-Ray effect. Chem. Phys. Lett. 2004, 397, 46-50.

(7) Jungwirth, P.; Tobias, D. J. Specific Ion Effects at the Air/Water Interface. Chem. Rev. 2006, 106, 1259-1281.

(8) Adamson, A. W.; Gast, A. P. Physical Chemistry of Surfaces; Wiley-Interscience: Sidney, Australia, 1997.

(9) Henry, C. L.; Parkinson, L.; Ralston, J. R.; Craig, V. S. J. A Mobile Gas-Water Interface in Electrolyte Solutions. J. Phys. Chem. C 2008, 112, 15094-15097.

(10) Long, F. A.; Nutting, G. C. The Relative Surface Tension of Potassium Chloride Solutions by a Differential Bubble Pressure Method1. J. Am. Chem. Soc. 1942, 64, 2476-2482.

(11) Petersen, P. B.; Saykally, R. J. Adsorption of Ions to the Surface of Dilute Electrolyte Solutions: The Jones-Ray Effect Revisited. J. Am. Chem. Soc. 2005, 127, 15446-15452.

(12) Passoth, G. Uber den Jones-Ray-effekt und die Oberflächenspannung verdünnter elektrolytlosungen. Z. Phys. Chem. 1959, 2110, 129-147.

(13) Priester, T.; Bartoszek, M.; Lunkenheimer, K. Influence of Surface-Active Trace Impurities on the Surface Properties of Aqueous Solutions of Oligoethylene Glycol Monooctyl Ethers. J. Colloid Interface Sci. 1998, 208, 6-13.

(14) Rosen, M. J. Purification of surfactants for studies of their fundamental surface properties. J. Colloid Interface Sci. 1981, 79, 587588.

(15) Miles, G. D.; Shedlovsky, L. Minima in Surface TensionConcentration Curves of Solutions of Sodium Alcohol Sulfates. J. Phys. Chem. 1944, 48, 57-62.

(16) Ward, R. N.; Davies, P. B.; Bain, C. D. Coadsorption of Sodium Dodecyl Sulfate and Dodecanol at a Hydrophobic Surface. J. Phys. Chem. B 1997, 101, 1594-1601. 
(17) Duignan, T. T.; Baer, M. D.; Mundy, C. J. Surfactant Impurities Can Explain the Jones-Ray Effect. 2018, ChemRxiv.org e-Print archive. DOI: $10.26434 /$ chemrxiv.5732976.v3.

(18) Uematsu, Y.; Bonthuis, D. J.; Netz, R. R. Charged SurfaceActive Impurities at Nanomolar Concentration Induce Jones-Ray Effect. J. Phys. Chem. Lett. 2018, 9, 189-193.

(19) Jones, G.; Ray, W. A. The surface tension of solutions of electrolytes as a function of the concentration I A differential method for measuring relative surface tension. J. Am. Chem. Soc. 1937, 59, 187-198.

(20) Chen, Y.; Okur, H. I.; Gomopoulos, N.; Macias-Romero, C.; Cremer, P. S.; Petersen, P. B.; Tocci, G.; Wilkins, D. M.; Liang, C.; Ceriotti, M.; et al. Electrolytes induce long-range orientational order and free energy changes in the H-bond network of bulk water. Sci. Adv. 2016, 2, e1501891.

(21) Okur, H. I.; Chen, Y.; Wilkins, D. M.; Roke, S. The Jones-Ray effect reinterpreted: Surface tension minima of low ionic strength electrolyte solutions are caused by electric field induced water-water correlations. Chem. Phys. Lett. 2017, 684, 433-442.

(22) Beattie, J. K.; Djerdjev, A. M.; Gray-Weale, A.; Kallay, N.; Lützenkirchen, J.; Preočanin, T.; Selmani, A. pH and the surface tension of water. J. Colloid Interface Sci. 2014, 422, 54-57.

(23) Wilkins, D. M.; Manolopoulos, D. E.; Roke, S.; Ceriotti, M. Communication: Mean-field theory of water-water correlations in electrolyte solutions. J. Chem. Phys. 2017, 146, 181103.

(24) Jarvis, N. L.; Scheiman, M. A. Surface potentials of aqueous electrolyte solutions. J. Phys. Chem. 1968, 72, 74-78.

(25) Gaines, G. L. Insoluble Monolayers at Liquid-Gas Interfaces; Wiley-Interscience: New York, 1966.

(26) Lunkenheimer, K.; Pergande, H. J.; Krüger, H. Apparatus for programmed high-performance purification of surfactant solutions. Rev. Sci. Instrum. 1987, 58, 2313-2316.

(27) Bewig, K. W.; Zisman, W. A. The Wetting of Gold and Platinum by Water. J. Phys. Chem. 1965, 69, 4238-4242. 